\title{
Anhang: Aufbau und Themen der Konjunkturberichte
}

Wie die „Hauptanstalt in Berlin“ entwickelte die Essener Abteilung ihre eigene Konjunkturstatistik, allerdings regional, für den rheinisch-westfälischen Industriebezirk. Als erstes Dokument, das die statistischen Materialien zusammenfasste und nach einheitlichen Gesichtspunkten verarbeitete, erschien 1928 das umfangreiche Handbuch „Wirtschaftszahlen Westen, Konjunkturstatistik des rheinisch-westfälischen Industriebezirks und Westdeutschlands“. Es wurde nicht wieder als eigenständiges Werk herausgegeben, sondern mit gesonderten Zusammenstellungen in den Konjunkturberichten fortgeführt. ${ }^{1}$ In diesen für die Öffentlichkeit bestimmten Konjunkturberichten des Essener Instituts erschienen von 1929 bis 1938/39 regelmäßig die Forschungsergebnisse der Abteilung Westen. ${ }^{2}$ Sie knüpften damit an die ersten bereits dargestellten Berichte an, die vorher unregelmäßig als Probehefte „nur einem engeren Kreis von Interessenten zugänglich gemacht“ worden waren. ${ }^{3}$ Das Erscheinen der „regelmäßigen Konjunkturberichte“ war im Februar 1929 für Däbritz der Anlass, den bisherigen Förderern der Abteilung Westen zu danken und anzukündigen, ihnen „diese Konjunkturberichte fortan kostenlos zu übersenden“. ${ }^{4}$

\section{Bibliografische Angaben}

Konjunkturberichte der Abteilung „Westen“, Essen, des Instituts für Konjunkturforschung, Berlin, I. - X. Jahrgang, 1929-1939, Berlin: Verlag von Reimar Hobbing 1929, ab dem VI. Jg. 1934, Hamburg: Hanseatische Verlagsanstalt.

Zur Abgrenzung des „Ruhrbezirks“ und des „rheinisch-westfälischen Industriebezirks“ siehe vor allem die Abhandlung im Doppelheft des Konjunkturberichts von

1 Siehe vor allem KB 1936/37, H. 3 (Vier Jahre Wirtschaftsaufstieg) und KB 1938/39, H. 1/2 (Wirtschaftszahlen „Westen“). Davor erschienen zudem kürzere tabellarische Übersichten: Wirtschaftszahlen „Westen“, KB 1929, H. 2, S. 22-27; KB 1930, H. 5, S. 18-23.

21929 u. 1930 je 6 Hefte, 19315 Hefte, 1932 u. 1933 je 4 Hefte, 19341 Heft u. 2/3 als Doppelheft, 1935 2 Hefte u. 3/4 als Doppelheft, 1936/37-1938/39 je 3 Hefte, davon 38/39 1/2 als Doppelheft. Beim IfK in Berlin war der erste Jahrgang der „Vierteljahrshefte zur Konjunkturforschung“ (VjhK) 1926 erschienen. Ausführlich dazu Krengel 1986, S. 14 ff.

3 KB 1937, H. 3 (Vier Jahre Wirtschaftsaufstieg), Vorwort von Däbritz S. V.

4 WWA K1 Nr. 571. Zu den Förderern „gehören der Rheinische Provinzialverband, Düsseldorf, die Stadtverwaltung Essen, der Verein für die bergbaulichen Interessen, Essen, die Nordwestliche Gruppe des Vereins Deutscher Eisen- und Stahlindustrieller, Düsseldorf, die Vereinigung von Banken und Bankiers in Rheinland und Westfalen, Köln, die Industrie- und Handelskammern Essen, Bochum, Dortmund und Duisburg." Über die IHK Bochum z.B. erhielt Däbritz eine Namensliste von 20 Abnehmern, welche die Konjunkturberichte direkt zugesandt bekamen. Siehe Briefwechsel zwischen Däbritz und der IHK Bochum 14. u. 20.2.1929, WWA K2 Nr. 259.

Ә OpenAccess. (c) 2018 Toni Pierenkemper, Rainer Fremdling, publiziert von De Gruyter. (cc))BY-NC-ND Dieses Werk ist lizenziert unter der Creative Commons Attribution-NonCommercial-NoDerivatives 4.0 Lizenz. 
1938/39. In dieser ausführlichen Erörterung wurde der Umfang bzw. die Abgrenzung des Ruhrbezirks aus seiner wirtschaftlichen Struktur, wie sie sich in den letzten hundert Jahren entwickelt hatte, abgeleitet. Nach den Analysen der Abteilung „Westen“ war „als das entscheidende Merkmal im Aufbau des Ruhrbezirks die hier vorhandene Schwerindustrie mit ihren beiden wichtigsten Pfeilern, dem Ruhrbergbau und der Eisen schaffenden Industrie anzusehen“. Für den wirtschaftlich definierten Ruhrbezirk wurde eine Verwaltungseinteilung auf Kreisebene gegeben. Zudem wurde die inhaltliche und räumliche Abgrenzung anderer (auch vom Essener Institut verwendeter) Regionalbezeichnungen, wie Ruhrgebiet, rheinisch-westfälischer Industriebezirk etc. diskutiert.

Die Konjunkturberichte behielten das inhaltliche Spektrum und ihren Aufbau im Wesentlichen bei. Lediglich die Sonderthemen variierten, manchmal beanspruchten sie ein ganzes Heft. Zudem war die allen Heften vorgeschaltete Gliederung nicht einheitlich und gleichermaßen ausführlich gestaltet. Üblich war die hier wiedergegebene Gliederung des IX. Jg. 1937/38 H. $2{ }^{6}$

\section{Konjunkturberichte}

IX. Jg. 1937/38 H. 2 Abgeschlossen Ende Februar 1938

Die Konjunkturlage im rheinisch-westfälischen Industriebezirk März 1938

Die Entwicklungstendenzen

I. Produktion und Absatz

1. Die Produktionsmittelindustrien

2. Der Baumarkt

3. Die Verbrauchsgüterindustrien

II. Der Arbeitseinsatz

III. Verkehr

IV. Preise, Löhne, Verbrauch

V. Finanzierungsfragen

Zusammenfassung

Die oben aufgeführten thematischen Schwerpunkte wurden regelmäßig behandelt. In die weit überwiegenden textlichen Erörterungen waren einzelne Grafiken und Tabellen eingebaut. Die Daten bezogen sich in der Regel auf monatliche Angaben. Jedem Heft war der Zeitpunkt (meistens der Monat) des Redaktionsschlusses zu entnehmen. Im Heft X. Jg. 1939 H. 3, abgeschlossen 31.05.1939, wurde erstaunlicherweise nicht angekündigt, dass es das „letzte“ war.

5 KB X. Jg. 1938/39 H. 1/2 S. 68-75. Die Abteilung Westen hatte zuvor schon mehrfach eine regionale Abgrenzung ihres Untersuchungsgegenstandes gegeben: siehe die Aufzählung ebd., S. 69.

6 Ganz ähnlich auch das beispielhaft angeführte Inhaltsverzeichnis bei Däbritz 1950, S. 29. 
Die im Mutterhaus seit 1926 erscheinenden „Vierteljahrshefte zur Konjunkturforschung“(VjhK) waren umfangreicher und behandelten neben der deutschen auch die „Konjunktur des Auslandes“. Sie veröffentlichten sogar wöchentliche Angaben. Nicht nur wegen der systematischen Auslandsberichterstattung war das Themenspektrum zudem vielfältiger als das Essener: z. B. wurden die „öffentliche Wirtschaft“, die „Landwirtschaft“ und „Außenhandel und Exportwirtschaft“ behandelt. ${ }^{7}$

Die folgende Tabelle stellt mit der Übersicht über alle Sonderthemen über die Standardberichterstattung der Konjunkturberichte hinaus das durchaus breite Forschungsspektrum des Essener Instituts bis Ende der 1930er Jahre dar.

7 Siehe z.B. VjhK 8. Jg. 1933. Ausführlich Krengel 1986, S. 12-19, S. 36 - 39. 


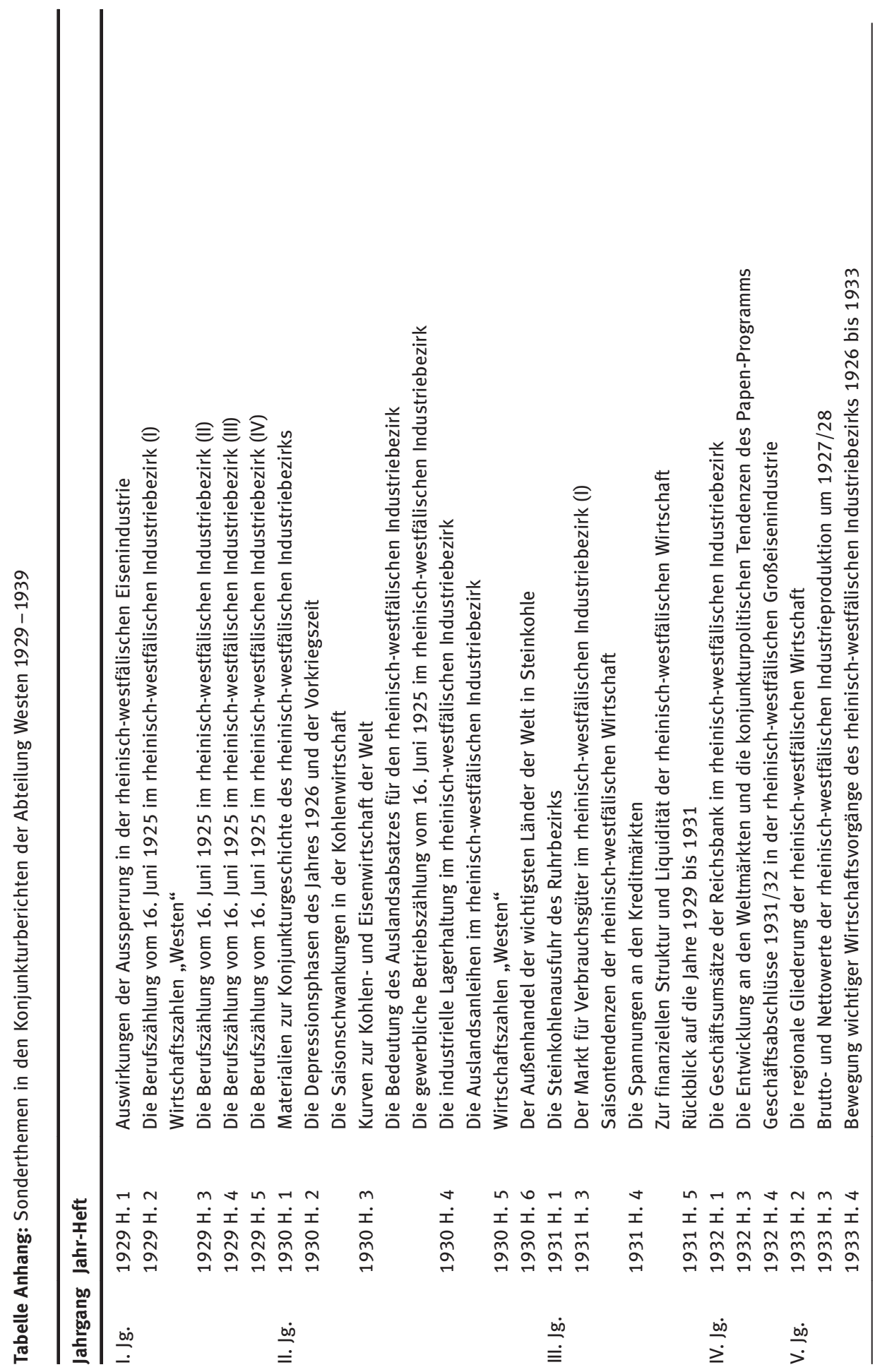




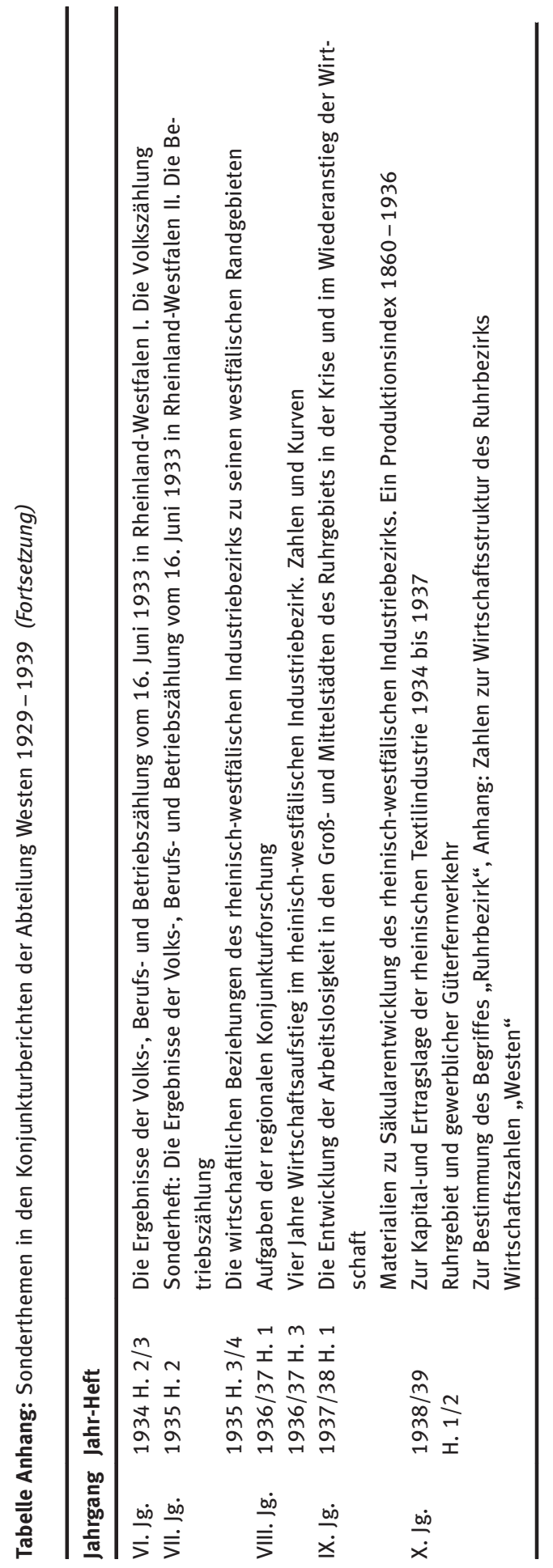

